\title{
Relação entre sentido de vida e satisfação com a vida
}

\author{
Leonardo de Oliveira Barros \\ Thaline da Cunha Moreira \\ Dianniffer Aparecida Oliveira \\ Ana Paula Porto Noronha
}

\section{RESUMO}

Esta pesquisa objetivou verificar a relação entre sentido de vida e satisfação com a vida e possíveis diferenças em razão de variáveis sociodemográficas em uma amostra de brasileiros. Participaram 901 sujeitos, 81,9\% do sexo feminino e com idade média de 35,19 anos. Foi aplicado um questionário semiaberto com o objetivo de realizar a avaliação do sentido existencial e a Escala de Satisfação com a Vida. A aplicação ocorreu de forma online e os dados foram tratados por meio de estatísticas descritivas e inferenciais. Foi possível observar que as pessoas que praticam alguma religião estão mais satisfeitas com a vida do que aquelas que não frequentam instituições religiosas. Em relação à escolaridade, indivíduos que declararam ter cursado Pós-Graduação apresentaram níveis mais altos de satisfação com a vida quando comparados com os que cursaram Ensino Médio e/ou Ensino Superior. Além disso, os homens afirmaram mais frequentemente ausência de algo importante na vida em comparação às muIheres. Os achados são discutidos à luz da literatura.

Palavras-chave: Psicologia positiva; Logoterapia; Bem-estar subjetivo

\section{ABSTRACT \\ Relationship between meaning of life and satisfaction with life}

This study aimed to verify the relationship between the meaning of life and satisfaction with life and possible differences due to sociodemographic variables in a sample of Brazilians. 901 subjects participated, $81.9 \%$ female and with an average age of 35.19 years. A semi-open questionnaire was applied in order to assess the existential meaning and the Life Satisfaction Scale. It was possible to observe that people who practice a religion are more satisfied with life than those who do not attend a religious institution. In relation to schooling, individuals who declared that they had completed a postgraduation course had higher levels of satisfaction with life than those who attended High School and Higher Education. In addition, men more often stated the absence of something important in life compared to women. The findings are discussed in light of the literature.

Keywords: Positive psychology; Logotherapy; Subjective well-being.

Sentido de vida (SV) refere-se à atribuição de um sentido incondicional e coerente para a existência, que promove a busca e realização de objetivos significativos e que aumenta os níveis de autorrealização e felicidade (Damásio et al., 2013a; Steger, 2009). 0 conceito é discutido inicialmente por Frankl $(1990,1991)$, que discorreu sobre o quanto a busca por um sentido para a vida pode ser considerada a motivação primária do ser humano, resultando em um comportamento de busca de valores. Tais valores podem ser de três tipos: vivenciais, criativos e atitudinais, implicando na capacidade de vivenciar algo ou alguém, criar ações sobre o mundo ou adotar uma postura em relação ao sofrimento inevitável (Frankl, 2011). Deste modo, ao longo do ciclo vital o homem irá se deparar com uma necessidade maior, qual

\section{Sobre os autores}

L. O. B. http://orcid.org/0000-00028406-0515

Universidade São Francisco Bragança Paulista, SP leonardobarros_lob@hotmail. com

T. C. M.

http://orcid.org/0000-0002-

6484-8583

Universidade São Francisco Bragança Paulista, SP thacmoreira@gmail.com

\section{A. 0.}

http://orcid.org/0000-00025536-2039

Universidade Anhanguera de Limeira - Limeira, SP

diannifferoli@gmail.com

\section{A. P. P. N.}

http://orcid.org/0000-0001-

6821-0299

Universidade São Francisco Bragança Paulista, SP ana.noronha@usf.edu.br

\section{Direitos Autorais}

Este é um artigo de acesso aberto e pode ser reproduzido livremente, distribuído, transmitido ou modificado, por qualquer pessoa desde que usado sem fins comerciais. 0 trabalho é disponibilizado sob a licença Creative Commons CC-BY-NC.

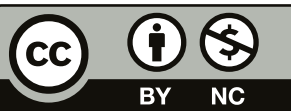




\section{H* INTERACÃO EM L PSICOLOGIA}

seja conferir sentido às experiências vivenciadas de modo a atribuir sentido por meio da transcendência de si mesmo para uma causa ou alguém.

0 engajamento da pessoa e a sua disponibilidade de se voltar para alguma causa ou alguém, é um fenômeno específico e individual (Frankl, 1993), que não é movido, a priori, pelo prazer ou felicidade, mas pode gerá-los (Frankl, 2003). Desta feita, o que o homem almeja necessariamente não é o prazer, mas a execução do objetivo que poderá conduzir para esse estado, uma vez que, se o SV fosse movido apenas pela satisfação pessoal, não teria pertinência. O sofrimento, ainda que seja evitado, faz parte da existência humana e consequentemente também é entendido como parte do SV (Silveira \& Mahfoud, 2008). Dessa forma, o propósito maior do ser humano é descobrir o sentido de sua vida, e com isso buscar sua satisfação pessoal e realização (Frankl, 1989).

A busca por um sentido de vida diminui com o passar dos anos, de acordo com Frankl (1991), uma vez que pessoas mais velhas tendem a perceber mais realizações em sua existência e atribuir sentidos reais em seus atos quando comparadas com pessoas mais novas (Aquino et al., 2017a). Todavia, o SV continua acessível também na velhice, uma vez que idosos ainda têm possibilidades de desenvolvimento à sua disposição (Vieira \& Aquino, 2016). Cabe ressaltar que na perspectiva da logoterapia, o tempo é ontológico, flui do futuro para o passado, não é fixo, sendo que o homem só completa sua essência na morte (Aquino et al., 2017b; Roehe, 2005). Além disso, ele poderá variar de acordo com as circunstâncias, sejam elas externas ou internas (Frankl, 1993; Silveira \& Mahfoud, 2008), não se caracterizando como um fenômeno constante, mas experimentado de formas distintas ao longo do desenvolvimento humano.

Ainda sobre os aspectos sociodemográficos e o SV, uma meta-análise identificou que a partir da meia idade há um declínio de sentido de vida e que o estado civil explicou $2,6 \%$ do SV, com maiores níveis para pessoas casadas (Pinquart, 2002). Em relação às diferenças por sexo, embora não haja um consenso na literatura sobre como estas se configuram (Damásio et al., 2013b; Martínez \& Castellanos, 2013; Pinquart, 2002), Noronha et al. (2018) identificaram que mulheres associam o SV à família enquanto homens o percebem como uma missão ou razão de viver. No entanto, entre pessoas com mais de 70 anos, as mulheres apresentaram níveis mais baixos de SV (Pinquart, 2002). Quanto à idade, Aquino et al. (2017a) encontraram resultados divergentes de Pinquart (2002), pois adultos (35 a 59) e idosos (60 a 87 anos) apresentam maior propósito de vida quando comparado com jovens (18 a 24 anos).
Leonardo de Oliveira Barros, Thaline da Cunha Moreira, Dianniffer Aparecida Oliveira e Ana Paula Porto Noronha
O SV é uma característica determinante da saúde mental e a sua perda pode estar associada ao abuso de drogas, depressão e suicídio (Kleiman \& Beaver, 2013; Volkert et al., 2017; Tan et al., 2018). Cabe ressaltar que a busca por um sentido pode não ser atendida, resultando em um uma sensação de vazio existencial ou ausência de conteúdo na vida (Frankl, 2011), sendo que nas sociedades pós-industriais há maiores ocorrências desses sentimentos em virtude da constante busca pelo ter, poder e prazer em detrimento da descoberta do sentido de vida (Aquino et al., 2010). Assim, investigações acerca do SV são importantes para auxiliar no entendimento de como estão sendo estabelecidas as buscas de sentido pela sociedade atual, assim como, permitir elaborar estratégias preventivas de saúde mental.

A busca por um sentido ou objetivo na vida é uma das principais fontes de motivação na vida das pessoas (Frankl, 1990). O SV também é parte do bem-estar, sendo que este é um conceito entendido a partir de duas perspectivas. A primeira é chamada de eudaimônica e está relacionada ao propósito e autorrealização, configurando-se como bem-estar psicológico, no qual se insere o SV (Damásio et al., 2013b; Delle Fave et al., 2011). A segunda perspectiva é a hedônica, relacionada a fatores externos e de satisfação, configurando-se como bem-estar subjetivo e que engloba a satisfação com a vida e os afetos negativos e positivos (Diener et al., 2002). Mais especificamente, a satisfação com a vida se refere à avaliação que o indivíduo pode fazer da sua vida, tanto de forma geral, como também particularmente em relação ao trabalho, amigos, família, saúde, entre outros (Reppold et al., 2019).

A satisfação com a vida se constitui como o componente cognitivo do bem-estar subjetivo (Diener \& Scollon, 2014). A este respeito, o estudo de Doğana et al. (2012), com estudantes universitários, indicou que a busca de sentido de vida prediz significativamente o bem-estar subjetivo, o que também foi evidenciado por Eryılmaz e Aypay (2011), com amostra de adolescentes. Mais recentemente, Souza (2020) encontrou que a presença de sentido de vida por ser explicada pelo bem-estar subjetivo em 17\%. Além disso, outras variáveis interferem no nível de percepção de bem-estar subjetivo, tais como sexo, idade, religiosidade e relacionamentos interpessoais (Casas et al., 2015; Silva et al., 2017).

Sentido de vida foi identificado como um potencial mediador entre religiosidade e bem-estar psicológico, entre religiosidade e satisfação com a vida, bem como entre autoestima e otimismo (Steger \& Frazier, 2005). De modo similar, o SV atua como preditor para o bem-estar psicológico e a qualidade de vida (Damásio, et al., 2013b). Sentido de vida, otimismo e bem-estar subjetivo também foram objeto de investigação de Ho et al. (2010) e os achados revelaram que os dois primeiros estão significativamente associados ao bem-estar. 


\section{H* INTERACÃO EM L* PSICOLOGIA}

Oishi e Diener (2013) examinaram a satisfação com a vida e o sentido de vida em 132 nações e encontraram que o último é maior naquelas mais pobres, o que no estudo foi explicado em razão da influência da maior religiosidade. No que diz respeito à renda, Ward e King (2016) concluíram que em níveis elevados de afetos positivos, a renda não estava relacionada com sentido de vida, mas quando havia baixo afetos positivos, o rendimento foi positivamente associado ao sentido de vida. Além disso, sentido de vida e adaptabilidade de carreira estão positivamente correlacionados (Yuen \& Yau, 2015).

Em que pese o fato de que o construto sentido de vida ter recebido maior atenção e ganhado mais legitimidade nos últimos anos, em razão da psicologia positiva e da investigação das características psicológicas positivas, suas pesquisas ainda carecem de incremento. Assim, torna-se importante verificar como se relacionam o construto se comporta a fim de fornecer maiores evidências de sua funcionalidade e subsidiar cientificamente nas intervenções. Ao considerar, também, a relevância dos construtos para a saúde mental (Kaler, 2006; Volkert et al., 2017; Tan et al., 2018), a presente pesquisa teve por objetivo verificar a relação entre o sentido de vida (por meio do levantamento do que as pessoas consideram mais importante em suas vidas) e a satisfação com a vida, bem como verificar diferenças em função de variáveis sociodemográficas.

\section{MÉTODO}

\section{PARTICIPANTES}

Esta pesquisa contou com uma amostra não-aleatória de conveniência. Participaram 901 brasileiros, sendo 81,9\% ( $n=738)$ do sexo feminino e $18,1 \%(n=163)$ do masculino, com idades variando entre 18 e 77 anos $(M=35,19 ; D P=11,9)$. Em relação ao nível de escolaridade, $17 \%(n=153)$ das pessoas declararam ter concluído o ensino médio, 36,9\% $(n=333)$ o ensino superior e $46,1 \%(n=415)$ concluiu pós-graduação. Em relação à ocupação no momento da pesquisa, $79,9 \%(n=720)$ afirmou estar trabalhando. Os participantes eram residentes de 25 estados brasileiros, com a maioria localizada no estado de São Paulo $(n=380)$ e em Minas Gerais $(n=150)$. Uma minoria da amostra foi composta por brasileiros que residiam em outros países $(n=13)$.

A atribuição religiosa dos participantes também foi levantada, sendo que metade da amostra se declarou católica $(n=452 ; 50,1 \%)$, seguidos dos que disseram ser espíritas ( $n=98 ; 10,9 \%)$; protestantes/evangélicos ( $n=72 ; 8 \%)$. Além desses, houve os que informaram ser cristãos sem especificar a denominação $(n=43 ; 4,8 \%)$; espiritualizados $(n=37$; $4,1 \%)$; ateus $(n=25 ; 2,8 \%)$; agnósticos $(n=17 ; 2,8 \%)$; umbandistas $(n=12 ; 1,3 \%)$ e os que acreditavam em Deus $(n=8 ; 0,9 \%)$.
Leonardo de Oliveira Barros, Thaline da Cunha Moreira, Dianniffer Aparecida Oliveira e Ana Paula Porto Noronha
Em menor frequência estavam, Candomblé, Budismo, Judaísmo, Wicca/Pagã, Testemunhas de Jeová, Teísmo, Messiânica, Hare Krishna e Islamismo (com um ou dois respondentes por religião/seita). Uma pequena parcela da amostra não respondeu à questão ( $n=3 ; 0,3 \%)$ e ainda houve a presença daqueles que disseram não ter uma religião $(n=109 ; 12,1 \%)$, ou não ter uma religião definida $(n=2 ; 0,2 \%)$.

\section{INSTRUMENTOS}

1- Escala de Satisfação com a Vida - ESV (Diener et al., 1985; adaptado para o português por Hutz et al., 2014): o instrumento é composto por cinco itens respondidos em escala tipo Likert de sete pontos, variando entre Discordo fortemente (1) e Concordo fortemente (7), sendo que os participantes devem marcar o grau de concordância com cada uma das afirmativas. Com a presente amostra, o alfa de Cronbach foi de 0,85 .

2- Questionário de avaliação do sentido existencial: foi desenvolvido pelos pesquisadores do presente estudo e buscou conhecer o sentido de vida. Além disso, foram identificados dados sociodemográficos, como idade, sexo, unidade de federação, escolaridade, situação laboral e religião. Para atender ao objetivo da pesquisa as seguintes questões foram elaboradas: "O que você considera mais importante em sua vida?" com intuito de identificar o que era entendido como o sentido da vida da pessoa, ou seja, aquilo para o que mais se devotavam (Frankl, 2011) e "Atribua uma nota de 0 a 10 para sua existência" com o intuito de mensurar percepção de satisfação com a vida. A primeira pergunta era aberta e sem limite de caracteres para a resposta.

\section{PROCEDIMENTOS}

O estudo está inserido em um projeto de pesquisa mais amplo, que foi devidamente apreciado e recebeu parecer favorável do Comitê de Ética em Pesquisa da Universidade São Francisco (CAAE: 20410713.3.0000.5514). Para a coleta de dados foi desenvolvido um protocolo na plataforma Google Drive, sendo disponibilizado o link nas redes sociais por um período de 10 dias durante o mês de junho de 2016. A primeira parte do questionário foi destinada a explicar os objetivos da pesquisa e o tempo de resposta necessário para completá-la. Após concordarem com o Termo de Consentimento Livre e Esclarecido (TCLE), os participantes tinham acesso ao questionário.

\section{PROCEDIMENTOS DE ANÁLISE DOS DADOS}

Inicialmente, os dados foram organizados por meio do programa estatístico SPSS v.20, tendo sido realizadas análises descritivas para a caracterização da amostra. Em seguida, foram empregadas análises de conteúdo para as perguntas 


\section{H. INTERACÃO EM PSICOLOGIA}

abertas do questionário, com o intuito de formar categorias de respostas. As categorias foram construídas com base nos pressupostos de Bardin (2004), tendo sido organizadas em unidades de respostas e a classificação das unidades em uma das categorias (Felicidade, Pessoas, Religião e Espiritualidade, Saúde, Trabalho ou Estudos, Relações Sociais, Ausência de algo importante e Outros) foi realizada de maneira independente por três juízes. A concordância entre eles foi de $100 \%$ em $99,1 \%$ das respostas, e em $0,89 \%$ foi de $66,7 \%$. As categorias serão descritas na seção resultados.

Por fim, empregaram-se análises estatísticas para comparação das médias dos participantes em relação às variáveis sexo, escolaridade, situação trabalhista e prática da religião. Utilizou-se correlação de Pearson, teste $t$ de Student, Análise de variância (ANOVA) e Prova de Tukey com o intuito de verificar diferenças estatisticamente significativas entre os grupos.

\section{RESULTADOS}

Em relação às respostas para a pergunta "O que você considera como mais importante em sua vida?" foram criadas oito categorias: Sentimento Positivos; Pessoas; Religião e Espiritualidade; Saúde; Trabalho ou Estudos; Relações Sociais; Ausência de algo importante e Outros. A categoria "Sentimentos Positivos" incluiu as respostas que se referiam a estar bem consigo e a sentimentos e afetos positivos (como exemplos, amor e alegria). A categoria "Pessoas" abordou as respostas referentes à família, pais, filhos ou companheiros. $\mathrm{Na}$ categoria "Religião e Espiritualidade" foram agrupadas as respostas que mencionavam Deus, servir à religião, evolução espiritual ou ser espiritualizado. Na categoria "Saúde" foram inseridas as respostas que abordavam questões como estar saudável, ter saúde, equilíbrio físico, entre outras.

A categoria "Trabalho e Estudos" reuniu as respostas que elencavam ter um trabalho, concluir a faculdade, ter sucesso financeiro ou profissional. A categoria "Relações sociais" agrupou aspectos como se dedicar ao próximo, ajudar o outro, cooperar para o bem da humanidade. Para a categoria "Ausência de algo importante" foram consideradas as respostas que apontavam não ter nada de importante na vida e, por fim, a categoria "Outros" reuniu as respostas que não foram possíveis de classificar em outras categorias como internet, lazer, entre outros. Após a categorização, procedeu-se à análise de concordância entre os juízes, tal como relatado anteriormente, e as comparações quanto às variáveis sociodemográficas, utilizando-se teste $t$ e ANOVA.

Em relação ao sexo, foram encontradas diferenças significativas apenas para a categoria "Ausência de algo importante" na qual os homens obtiveram maiores pontuações quando comparados às mulheres $(p<0,05)$. Quando comparadas as
Leonardo de Oliveira Barros, Thaline da Cunha Moreira, Dianniffer Aparecida Oliveira e Ana Paula Porto Noronha respostas em razão da pessoa praticar ou não sua religião, foram encontradas diferenças com significância estatística apenas para a categoria "Religião e Espiritualidade", sendo que as pessoas que praticam alguma religião endossaram mais a categoria $(p<0,001)$. Ao comparar as variáveis situação trabalhista (estar trabalhando ou não) e nível de escolaridade, não foram encontradas diferenças significativas.

Dando continuidade às análises, realizou-se teste $t$ e ANOVA entre o escore total na Escala de Satisfação com a Vida e as variáveis sociodemográficas. Na comparação entre os sexos, as mulheres obtiveram maiores pontuações quando comparadas aos homens $(p<0,05)$. Além disso, as pessoas que praticam alguma religião indicaram estar mais satisfeitas com a vida do que as que não frequentam uma instituição religiosa $(p<0,05)$. Ao considerar o grau de escolaridade, indivíduos que declararam ter cursado pós-graduação apresentaram níveis mais altos de satisfação com a vida quando comparados com os que cursaram ensino médio e superior, tal como pode ser observado na Tabela 1. Não foram encontrados resultados significativos para as demais variáveis.

\section{Tabela 1. Análise de variância e Prova de Tukey entre escolaridade e escore geral na ESV}

\begin{tabular}{|c|c|c|c|c|c|c|}
\hline \multirow{4}{*}{$\begin{array}{c}\text { Escore de } \\
\text { Satisfação } \\
\text { com a } \\
\text { Vida }\end{array}$} & \multirow{2}{*}{ gl } & \multirow{2}{*}{$F$} & \multirow{2}{*}{$\mathrm{n}$} & \multirow{2}{*}{ Escolaridade } & \multicolumn{2}{|c|}{ Subconjuntos } \\
\hline & & & & & 1 & 2 \\
\hline & 2 & $7,035^{\star *}$ & 333 & Ensino Superior & 25,33 & \\
\hline & & & 153 & Ensino Médio & 25,55 & \\
\hline & & & 415 & Pós-Graduação & & 26,85 \\
\hline
\end{tabular}

$p<0,001$

$\mathrm{Na}$ sequência, foram recodificados os escores por itens (somatória das pontuações possíveis em cada item) nas respostas à Escala de Satisfação com a Vida, gerando três quartis para as pontuações; quartil 1 (menor que 25, pontuação baixa), quartil 2 (entre 25 e 75, pontuação média) e quartil 3 (acima de 75 , pontuação alta). Posteriormente, buscaram-se diferenças de médias em relação às categorias qualitativas considerando a nova organização e os resultados significativos foram encontrados apenas para o item "se eu pudesse viver uma segunda vez, não mudaria quase nada na minha vida", tal como apresentado na Tabela 2.

Observa-se que houve diferenciação entre grupos formando dois subconjuntos nas duas categorias que apresentaram resultados estatisticamente significativos. Pessoas que consideram a religião ou espiritualidade como algo mais importante da vida endossaram mais a afirmação "se eu pudesse viver uma segunda vez não mudaria quase nada" com pontuações médias ou altas. 
Tabela 2. Análise de variância e Prova de Tukey entre os itens da ESV e as categorias qualitativas

\begin{tabular}{|c|c|c|c|c|c|c|}
\hline \multirow{2}{*}{ Categoria } & \multirow{2}{*}{ gl } & \multirow{2}{*}{$F$} & \multirow{2}{*}{$\mathrm{n}$} & \multirow{2}{*}{ Item 5} & \multicolumn{2}{|c|}{ Subconjuntos } \\
\hline & & & & & 1 & 2 \\
\hline \multirow{3}{*}{$\begin{array}{c}\text { Religião e } \\
\text { espiritualidade }\end{array}$} & 2 & $5,010^{* *}$ & 266 & Baixo & 0,07 & \\
\hline & & & 280 & Médio & & 0,14 \\
\hline & & & 355 & Alto & & 0,15 \\
\hline \multirow{3}{*}{$\begin{array}{l}\text { Relações } \\
\text { sociais }\end{array}$} & 2 & $5,851^{\star *}$ & 266 & Baixo & 0,05 & \\
\hline & & & 355 & Médio & 0,06 & \\
\hline & & & 280 & Alto & & 0,12 \\
\hline
\end{tabular}

$* \star p<0,001$

Além desse grupo, pessoas que valorizam mais as relações sociais também se destacaram nesse item, havendo diferenciação de uma parte da amostra com pontuações mais altas. Esse resultado indica que indivíduos que percebem a dimensão espiritual ou as relações próximas como aspecto mais importante, tendem a estar mais satisfeitos com a vida a ponto de não desejarem mudar nada, caso fosse possível.

Na sequência, analisou-se se a idade se relaciona à percepção de satisfação com a vida. Dada a variabilidade de idades, elas foram agrupadas em quartis, compondo três grupos. 0 grupo 1 reuniu os participantes com idade até 26 anos, o grupo 2 contemplou aqueles com idade entre 27 e 43 anos e o grupo 3 os participantes com idade a partir de 44 anos. Os resultados são apresentados na Tabela 3 .

Os resultados foram todos estatisticamente significativos e evidenciam que as pessoas com maior idade apresentaram maiores médias nos itens da ESV diferenciando-se das mais jovens. Posteriormente, buscou-se verificar a relação entre a nota atribuída para a vida e os resultados na ESV, sendo que para isso as notas foram organizadas em percentis, de modo que no grupo 1 ficaram aqueles que atribuíram nota de 0 a 6 , no grupo 2 foram reunidos os que atribuíram nota de 7 a 8 e, por fim, no último, os que atribuíram 9 ou 10 . 0 agrupamento também se deu quanto aos quartis: menor que 25 , entre 25 e 75 e acima de 75 . Realizou-se a ANOVA e a prova de Tukey e os resultados são apresentados na Tabela 4.

Verifica-se que houve diferença significativa entre as notas da vida em relação aos itens da Escala de Satisfação com a Vida. Nesse sentido, pessoas que atribuíram menores notas para a vida apresentaram menores pontuações quando comparadas com as que atribuíram maior nota para a vida, sendo que estas últimas apresentaram maiores pontuações em todos os itens da ESV.
Tabela 3. Análise de variância e Prova de Tukey entre os itens da ESV e idade

\begin{tabular}{|c|c|c|c|c|c|c|}
\hline \multirow{2}{*}{ Item } & \multirow{2}{*}{ gl } & & \multirow{2}{*}{$\mathrm{n}$} & \multirow{2}{*}{$\begin{array}{l}\text { Grupo } \\
\text { etário }\end{array}$} & \multicolumn{2}{|c|}{ Subconjuntos } \\
\hline & & & & & 1 & 2 \\
\hline \multirow{3}{*}{$\begin{array}{l}\text { 1- Na maioria dos } \\
\text { aspectos, minha } \\
\text { vida é próxima ao } \\
\text { meu ideal }\end{array}$} & 2 & $13,254^{\star *}$ & 244 & 1 & 4,914 & \\
\hline & & & 428 & 2 & 5,070 & \\
\hline & & & 228 & 3 & & 5,522 \\
\hline \multirow{3}{*}{$\begin{array}{l}\text { 2- As condições } \\
\text { da minha vida são } \\
\text { excelentes }\end{array}$} & 2 & $7,694^{* *}$ & 244 & 1 & 5,090 & \\
\hline & & & 428 & 2 & 5,199 & \\
\hline & & & 228 & 3 & & 5,544 \\
\hline \multirow{3}{*}{$\begin{array}{l}3 \text { - Estou satisfeito } \\
\text { com minha vida }\end{array}$} & 2 & $11,991^{\text {** }}$ & 244 & 1 & 5,045 & \\
\hline & & & 428 & 2 & 5,100 & \\
\hline & & & 228 & 3 & & 5,579 \\
\hline \multirow{3}{*}{$\begin{array}{l}4 \text { - Dentro do } \\
\text { possível tenho as } \\
\text { coisas importantes } \\
\text { que quero em } \\
\text { minha vida }\end{array}$} & 2 & $3,796^{\star \star}$ & 428 & 2 & 5,783 & \\
\hline & & & 244 & 1 & 5,811 & 5,811 \\
\hline & & & 228 & 3 & & 6,053 \\
\hline \multirow{3}{*}{$\begin{array}{l}5 \text { - Se eu pudesse } \\
\text { viver uma segunda } \\
\text { vez, não mudaria } \\
\text { quase nada na } \\
\text { minha vida }\end{array}$} & 2 & $3,458^{* *}$ & 244 & 1 & 4,484 & \\
\hline & & & 428 & 2 & 4,505 & 4,505 \\
\hline & & & 228 & 3 & & 4,882 \\
\hline
\end{tabular}

$* \star p<0,001$

Tabela 4. Análise de variância e Prova de Tukey entre os itens da ESV e a nota da vida

\begin{tabular}{|c|c|c|c|c|c|c|c|}
\hline \multirow{2}{*}{ Item } & \multirow{2}{*}{ gl } & & \multirow{2}{*}{$\mathrm{N}$} & \multirow{2}{*}{$\begin{array}{l}\text { Percentil } \\
\text { - Nota da } \\
\text { vida }\end{array}$} & \multicolumn{3}{|c|}{ Subconjuntos } \\
\hline & & & & & 1 & 2 & 3 \\
\hline \multirow{3}{*}{$\begin{array}{l}1 \text { - Na maioria dos } \\
\text { aspectos, minha } \\
\text { vida é próxima ao } \\
\text { meu ideal }\end{array}$} & 2 & $174,77^{\star \star}$ & 326 & Baixo & 4,26 & & \\
\hline & & & 296 & Médio & & 5,30 & \\
\hline & & & 279 & Alto & & & 6,01 \\
\hline \multirow{3}{*}{$\begin{array}{l}2 \text { - As condições da } \\
\text { minha vida são } \\
\text { excelentes }\end{array}$} & 2 & $111,17^{* *}$ & 326 & Baixo & 4,55 & & \\
\hline & & & 296 & Médio & & 5,35 & \\
\hline & & & 279 & Alto & & & 5,99 \\
\hline \multirow{3}{*}{$\begin{array}{l}3 \text { - Estou satisfeito } \\
\text { com minha vida }\end{array}$} & 2 & $239,36^{\star *}$ & 326 & Baixo & 4,22 & & \\
\hline & & & 296 & Médio & & 6,43 & \\
\hline & & & 279 & Alto & & & 6,13 \\
\hline \multirow{3}{*}{$\begin{array}{l}4 \text { - Dentro do } \\
\text { possível tenho as } \\
\text { coisas importantes } \\
\text { que quero em } \\
\text { minha vida }\end{array}$} & 2 & $101,53^{* \star}$ & 326 & Baixo & 5,21 & & \\
\hline & & & 296 & Médio & & 5,98 & \\
\hline & & & 279 & Alto & & & 6,49 \\
\hline \multirow{3}{*}{$\begin{array}{l}5 \text { - Se eu pudesse } \\
\text { viver uma segunda } \\
\text { vez, não mudaria } \\
\text { quase nada na } \\
\text { minha vida }\end{array}$} & 2 & $138,49^{* *}$ & 326 & Baixo & 3,47 & & \\
\hline & & & 296 & Médio & & 4,77 & \\
\hline & & & 279 & Alto & & & 5,72 \\
\hline
\end{tabular}

$* * p<0,001$ 


\section{H* INTERACÃO EM L PSICOLOGIA}

\section{DISCUSSÃO}

Esta pesquisa teve como objetivo verificar as relações entre sentido de vida e a satisfação com a vida como um dos elementos do bem-estar subjetivo em uma amostra de adultos e investigar possíveis diferenças nos construtos em razão de variáveis demográficas. Em relação às diferenças encontradas em função das variáveis sociodemográficas para as categorias de sentido de vida, os resultados na comparação entre sexos não corroboram os achados da literatura (Damásio et al., 2013a; Martínez \& Castellanos, 2013), que versam sobre a inexistência de diferenças. Na presente pesquisa, os homens endossaram mais a categoria "Ausência de algo importante". A este respeito, a título de hipótese, pode-se aventar que culturalmente homens são menos propensos a refletir sobre sua existência e a expressar seus afetos. Assim, eles não seriam estimulados a pensar sobre o sentido de suas vidas (Baliscei et al., 2015), o que pode levá-los a essa percepção de vazio existencial ou ausência de conteúdo na vida (Frankl, 2011).

Em outra medida, as pessoas adeptas a alguma religião entenderam a "Religiosidade e a Espiritualidade" como os elementos mais importantes em suas vidas, o que está em concordância com os achados de Steger e Frazier (2005) e Oishi e Diener (2013), que notaram em seus estudos o papel mediador e influenciador da religiosidade na percepção de ter um sentido de vida. Em acréscimo, conforme indica Frankl (2003) a religiosidade apresenta-se como uma fonte de sentido de vida por responder às questões existenciais e para permitir que haja a transcendência, permitindo que uma pessoa se integre de forma bio-psico-socio-espiritual e torne-se verdadeiramente humana.

Ao se analisar as pontuações dos participantes na ESV, nota-se que aqueles que praticam a religião pontuam mais alto na escala, demonstrando mais satisfação com suas vidas. Assim, a prática e presença da religião parece contribuir tanto para a presença de um sentido de vida como para a satisfação com ela. A espiritualidade - com ou sem religião - pode ser uma das possibilidades de busca por sentido (Silva et al., 2020). Além disso, a religiosidade influencia positivamente os níveis de satisfação com a vida e pode atuar como um fator protetivo e promotor de saúde mental (Fleury et al., 2018).

Esses dados foram congruentes com as análises das diferenças a partir da recodificação dos quartis (baixo, médio, alto) nos itens da ESV, em que os resultados significativos em duas categorias de sentido de vida no item "Se eu pudesse viver uma segunda vez, não mudaria quase nada na minha vida" dão dois indicativos. 0 primeiro é em relação a categoria "Religião e Espiritualidade" no qual as pessoas com menores pontuações diferenciaram-se dos demais, na mesma perspectiva já discutida anteriormente, em que assim como
Leonardo de Oliveira Barros, Thaline da Cunha Moreira, Dianniffer Aparecida Oliveira e Ana Paula Porto Noronha a prática da religião pode representar a existência de um sentido de vida, a ausência desta religiosidade pode indicar uma insatisfação e leva-los a não perceberem um sentido pra ela (Steger \& Frazier, 2005).

A segunda categoria que obteve relevância estatística foi "Relações sociais", na qual pessoas com altas pontuações no item se diferenciaram dos demais. Nesse sentido, ao considerar que esta categoria reuniu respostas relativas a ações altruístas, o resultado está em consonância com o que propõe Frankl (1993) de que o sentido de vida pode ser encontrado a partir da dedicação e serviço por uma causa ou alguém. A presença destas ações altruístas e das relações sociais que levam a ter um sentido de vida ainda são importantes para a saúde mental das pessoas, pois a deficiência e ausência destas ações pode favorecer a presença de depressão, suicídio e abuso de drogas (Kleiman \& Beaver, 2013; Volkert et al., 2017; Tan et al., 2018).

A comparação entre as notas atribuídas para a vida e as médias nos itens da ESV, apresentou uma formação de subconjuntos coerente. Os dados encontrados indicam que as pessoas que atribuíram maiores notas para sua vida obtiveram maiores pontuações nos itens da ESV, ou seja, afirmaram maior satisfação com suas vidas. Tal resultado corrobora da satisfação percebida após a autoavaliação global da própria vida enquanto elemento que pode gerar bem-estar subjetivo (Diener et al., 2002). Entretanto, percebe-se que no estudo de Ward e King (2016) os afetos positivos (também componentes do bem-estar subjetivo) estavam desassociados ao sentido de vida. Desta forma, nota-se o quanto ainda é necessário investigar mais afundo a relação entre estes construtos e, consequentemente, aprofundar a discussão a partir das concepções eudaimônica e hedonista do bem-estar.

No que se refere às pontuações na ESV, verificou-se que as mulheres apresentaram as maiores pontuações, demonstrando estarem mais satisfeitas com suas vidas. Na mesma direção, as pessoas com uma formação superior mais avançada (Pós-graduação) e as mais velhas também apresentaram estarem mais satisfeitas com a vida. Estes dados corroboram os achados de Doğana et al. (2012) e Damásio et al. (2013a), que notaram em estudantes universitários e professores que a busca de sentido de vida prediz o bem-estar subjetivo (composto pela satisfação com a vida) e, o estudo de Aquino et al. (2017a) que identificaram que os idosos possuem mais propósitos de vida. A partir destes dados pode-se verificar que de fato são diversas as variáveis que interferem na satisfação com a vida e consequentemente na percepção de sentido na vida, assim como colocado por Casas et al. (2015) e Silva et al. (2017). 


\section{H MTERAC̄OOEM ET PSICOLOGIA}

\section{CONSIDERAÇÕES FINAIS}

Considerando a importância de aprofundar os conhecimentos sobre os construtos sentido de vida e satisfação com a vida e dos aspectos adaptativos do ser humano que podem contribuir para as intervenções psicológicas (Kaler, 2006), ao analisar a relação entre estes construtos, percebeu-se, neste estudo, que a religião/espiritualidade e a felicidade são variáveis bastante relevantes. Assim, estas dimensões precisam estar presentes nas agendas de pesquisa e nos programas de intervenção das psicólogas e psicólogos nos vários contextos profissionais. Tal fato não implica ter-se uma psicologia religiosa ou espiritualista, mas sim que tais aspectos não sejam ignorados quando da análise da constituição dos fenômenos humanos.

Além disso, reforça-se a necessidade de a psicologia começar a se atentar aos aspectos saudáveis do ser humano, atuando de forma preventiva e potencializando as capacidades e não focando apenas nas psicopatologias. Nesse sentido, compreender o sentido de vida, bem como satisfação com a vida podem ser estratégias adequadas para o delineamento de intervenções que visem estimular o desenvolvimento dos aspectos mais adaptativos do indivíduo.

Apesar das contribuições, este estudo apresenta limitações em relação à amostra que além de ser composta apenas por adultos também teve maior concentração de participantes oriundos do estado de São Paulo, não permitindo buscar diferenças em função da região geográfica, fato que pode ser pauta de futuras pesquisas que considerem o impacto dos aspectos culturais e locais nos construtos. De todo modo, este estudo não pretende esgotar as discussões sobre o assunto e nem determinar a ocorrência dos fatos, assim, sugerem-se novos estudos que agreguem também os adolescentes e que associem o sentido de vida com outras variáveis da psicologia positiva, tais como projeto de vida, afetos positivos e negativos e bem-estar subjetivo, pois, estes podem ter estreita relação com o construto sentido de vida.

\section{DECLARAÇÃO DA CONTRIBUIÇÃO DOS AUTORES:}

Certificamos que todos os autores participaram suficientemente do trabalho para tornar pública sua responsabilidade pelo conteúdo. A contribuição de cada autor pode ser atribuída como se segue:

LOB contribuiu com a investigação, metodologia, análise formal de dados, redação (rascunho) e visualização. TCM e DAO contribuíram com a conceitualização, investigação, metodologia e redação (rascunho). APPN contribuiu com a administração do projeto, metodologia, investigação e redação (revisão e edição).
Leonardo de Oliveira Barros, Thaline da Cunha Moreira, Dianniffer Aparecida Oliveira e Ana Paula Porto Noronha

\section{DECLARAÇÃO DE CONFLITOS DE INTERESSE}

Os autores declaram que não há conflitos de interesse neste artigo.

\section{DECLARAÇÃO DE FINANCIAMENTO}

A pesquisa relatada no manuscrito foi financiada pelos próprios autores.

\section{REFERÊNCIAS}

Aquino, T. A. A., Alves, A. C. D., de Aguiar, A. A., \& de Oliveira Refosco, R. F. (2010). Sentido da vida e conceito de morte em estudantes universitários: um estudo correlacional. Interação em Psicologia, 14(2), 233-243. https://revistas. ufpr.br/psicologia/article/view/16696/13924

Aquino, T. A. A., Gouveia, V. V., Gomes, E. S., \& Sá, L. B. M. (2017a). La percepción de sentido de la vida en el ciclo vital: una perspectiva temporal. Avances em Psicología Latino americana, 35(2), 375-386. https://doi.org/10.12804/ revistas.urosario.edu.co/apl/a.3728

Aquino, T. A. A., Serafım, T. D. B., Silva, H. D. M., Barbosa, E. L., Cirne, E. A., Ferreira, F. R., \& Dantas, P. R. S. (2017b). Visões de morte, ansiedade e sentido da vida: um estudo correlacional. Psicologia Argumento, 28(63), 289-302. https://periodicos.pucpr.br/index.php/psicologiaargumento/article/ view/20069/19351

Baliscei, J. P., Teruya, T. K., \& Stein, V. (2015). Como ser "homem"? Investigando discursos sobre masculinidades. Revista Digital do LAV, 8(1), 88-104. http://www.redalyc.org/ articulo. 0 a id $=337038443006$

Bardin, L. (2004). Análise de conteúdo (3nd ed.). Edições 70.

Casas, F., Sarriera, J. C., Alfaro, J., González, M., Bedin, L., Abs, D., Figuer, C., \& Valdenegro, B. (2015). Reconsidering life domains that contribute to subjective well-being among adolescents with data from three countries. Journal of Happiness Studies, 16(2), 491-513. https://doi.org/10.1007/ s10902-014-9520-9

Damásio, B. F., Koller, S. H., \& Schnell, T. (2013a). Sources of Meaning and Meaning in Life Questionnaire (SoMe): Psychometric Properties and Sociodemographic Findings in a Large Brazilian Sample. Acta de Investigación Psicológica, 3(3), 1205-1228. http://dx.doi.org/10.1016/ S2007-4719(13)70961-X

Damásio, B. F., Melo, R. L. P., \& Silva, J. P. (2013b). Sentido de Vida, Bem-Estar Psicológico e Qualidade de Vida em Professores Escolares. Paidéia, 23(54), 73-82. https://doi. org/10.1590/1982-43272354201309 
Delle Fave, A., Brdar, I., Freire, T., Vella-Brodrick, D., \& Wissing M. (2011). The eudaimonic and hedonic components of happiness: qualitative and quantitative findings. Social Indicators Research, 100(2), 185-207. https://doi. org/10.1007/s11205-010-9632-5

Diener, E., Emmons, R. A., Larsen, R. J., \& Griffin, S. (1985). The satisfaction with life scale. Journal of Personality Assessment, 49(1), 71-75. https://doi.org/10.1207/s15327752jpa4901_13

Diener, E., Lucas, E. R., \& Oishi, S. (2002). Subjective Well-Being - The Science of Hapiness and Life Satisfaction. In C. R. Snyder, \& S. J. Lopez (Eds.), Handbook of Positive Psychology (pp. 63-73). Oxford University Press.

Diener, E., \& Scollon, C. (2014). The what, why, when, and how of teaching the science of subjective well-being. Teaching of Psychology, 41, 175-183. https://doi. org/10.1177/0098628314530346

Doğana,T., Sapmaz, F., Telb, F. D., Sapmazc, S., \& Temizelc, S. (2012). Meaning in Life and Subjective Well-Being among Turkish University Students. Procedia - Social and Behavioral Sciences, 55, 612-617. https://doi.org/10.1016/j.sbspro.2012.09.543

Eryılmaz, A., \& Aypay, A. (2011). Investigation of relationship between adolescents' subjective wellbeing and identity status. University of Dicle Journal of Ziya Gökalp Education Faculty, 16, 167-179. https://dergipark.org.tr/tr/download/ article-file/787063

Fleury, L. F. O., Gomes, A. M. T., Rocha, J. C. C. C., Formiga, N. S., Tavares e Souza, M. M., Marques, S. C., \& Bernardes, M. M. R. (2018). Religiosidade, estratégias de coping e satisfação com a vida: Verificação de um modelo de influência em estudantes universitários. Revista Portuguesa de Enfermagem de Saúde Mental, (20), 51-57. https://doi. org/10.19131/rpesm.0226

Frankl, V. E. (1989). Psicoterapia e sentido da vida. Quadrante.

Frankl, V. (1990). Logoterapia y análisis existencial. Herder.

Frankl, V. E. (1991). Em busca de sentido: um psicólogo no campo de concretação (W. O. Schlupp \& C. C. Aveline, Trad.). Vozes.

Frankl, V. E. (1993). A presença ignorada de Deus (3a ed.). Vozes.

Frankl, V. E. (2003). Psicoterapia e sentido da vida (A. M. Castro, Trad.). Quadrante.

Frankl, V. E. (2011). A vontade de sentido. Fundamentos e aplicações da Logoterapia (I. S. Pereira, Trad.). Paulus.

Ho, M. Y., Cheung, F. M., \& Cheung, S. F. (2010). The role of meaning in life and optismim in promoting well-being. Personality and Individual Differences, 48, 658-663. https://doi. org/10.1016/j.paid.2010.01.008

Hutz, C. S., Zanon, C., \& Bardagi, M. P. (2014). Satisfação de Vida. In C. S. Hutz (Ed.), Avaliação em Psicologia Positiva (pp. 43-48). Artmed.
Kaler, M. (2006). The Meaning in Life Questionnaire: Assessing the Presence of and Search for Meaning in Life. Journal of Counseling Psychology, 53(1), 80-93. https://doi. org/10.1037/0022-0167.53.1.80

Kleiman, E. M., \& Beaver, J. B. (2013). A meaningful life is worth living: Meaning in life as a suicide resiliency factor. Psychiatry Research, 210, 934-939. https://doi.org/10.1016/j. psychres.2013.08.002

Martínez, E., \& Castellanos, C. (2013). Percepción de sentido de vida en universitarios colombianos. Pensamiento Psicologico, 11(1), 71-82. http://www.redalyc.org/ pdf/801/80127000009.pdf

Noronha, A. P. P., Oliveira, D. A., Barros, L. O., \& Moreira, T. C. (2018). Variáveis associadas ao sentido de vida. Revista da Abordagem Gestáltica, 24(1), 35-43. https://doi. org/10.18065/RAG.2018v24n1.4

Oishi, S., \& Diener, E. (2013). Residents of Poor Nations Have a Greater Sense of Meaning in Life Than Residents of Wealthy Nations. Psychological Science, 25(2), 422-430. https://doi.org/10.1177/0956797613507286

Pinquart, M. (2002). Creating and maintaining purpose in life in old age: A meta-analysis. Ageing International, 27(2), 90114. https://doi.org/10.1007/s12126-002-1004-2

Reppold, C., Kaiser, V., Zanon, C., Hutz, C., Casanova, J. R., \& Almeida, L. S. (2019). Escala de Satisfação com a Vida: Evidências de validade e precisão junto de universitários portugueses. Revista de Estudios e Investigación em Psicología y Educación, 6(1), 15-23. https://doi.org/10.17979/ reipe.2019.6.1.4617

Roehe, M. V. (2005). Revendo idéias de Viktor Frankl no centenário de seu nascimento. PSICO, 36(3), 311-314. https:// dialnet.unirioja.es/servlet/articulo?codigo $=5161603$

Silva, D. G. D., Giordani, J. P., \& Dell'Aglio, D. D. (2017). Relações entre satisfação com a vida, com a família e com as amizades e religiosidade na adolescência. Estudos Interdisciplinares em Psicologia, 8(1), 38-54. https://doi.or$\mathrm{g} / 10.5433 / 2236-6407.2016 \mathrm{v} 8 \mathrm{n} 1 \mathrm{p} 38$

Silva, A. B. S., Guerra, V. M., Pirola, G. P., Galvão, J. A., \& Zanotelli, L. G. (2020). Relação entre sentido de vida e espiritualidade na América Latina: uma revisão integrativa da literatura. Interação em Psicologia, 24(2), 215-229. https:// doi.org/10.5380/psi.v24i2.66020

Silveira, D. R., \& Mahfoud, M. (2008). Contribuições de Viktor Emil Frankl ao conceito de resiliência. Estudos de Psicologia, 25(4), 567-576. https://doi.org/10.1590/S0103166X2008000400011

Souza, M. H. (2020). Relações entre bem-estar subjetivo e sentido de vida em estudantes universitários [Dissertação de Mestrado, Universidade São Francisco].

Steger, M. F. (2009). Meaning in life [Sentido na Vida]. In S. J. Lopez (Ed.), Oxford handbook of positive psychology (2nd ed., pp. 679-687). Oxford University Press. 


\section{- IN INERACÃO EM LFICOLOGIA}

Steger, M. F., \& Frazier, P. (2005). Meaning in life: One link in the chain from religiousness to well-being. Journal of Counseling Psychology, 52(4), 574-582. https://doi. org/10.1037/0022-0167.52.4.574

Tan, L., Chen, J., Xia, T., \& Hu, J. (2018). Predictors of Suicidal Ideation Among Children and Adolescents: Roles of Mental Health Status and Meaning in Life. Child \& Youth Care Forum, 47(2), 219-231. https://doi.org/10.1007/s10566017-9427-9

Vieira, D. C. R., \& Aquino, T. A. A. (2016). Vitalidade Subjetiva, Sentido na Vida e Religiosidade em Idosos: Um Estudo Correlacional. Temas em Psicologia, 2(24), 483-494. https://doi.org/10.9788/TP2016.2-05Pt

Volkert, J., Härter, M., Dehoust, M. C., Ausín, B., Canuto, A., Da Ronch, C., Sulling, A., Grassi, L., Munoz, M., Santos-Olmo, A. B., Sehner, S., Weber, K., Wegscheider, K., Wittchen, H. U., Schulz, H., \& Andreas, S. (2017). The role of meaning in life in community-dwelling older adults with depression and
Leonardo de Oliveira Barros, Thaline da Cunha Moreira, Dianniffer Aparecida Oliveira e Ana Paula Porto Noronha

relationship to other risk factors. Aging \& Mental Health, 1-7. https://doi.org/10.1080/13607863.2017.1396576

Ward, S. J., \& King, L. A. (2016). Poor but Happy? Income, Happiness, and Experienced and Expected Meaning in Life. Social Psychological and Personality Science, 7(5), 463470. https://doi.org/10.1177/1948550615627865

Yuen, M., \& Yau, J. (2015). Relation of career adaptability to meaning in life and connectedness among adolescentes in Hong Kong. Journal of Vocational Behavior, 91, 147-156. https://doi.org/10.1016/j.jvb.2015.10.003

Data de submissão: $29 / 08 / 2019$ Primeira decisão editorial: 29/10/2020 Aceite em: 03/12/2020 